\title{
Generation of Two-Dimensional Grammar Based Pattern Languages using Array Rewriting P System
}

\author{
Christopher Kezia Parimalam, J.D.Emerald
}

\begin{abstract}
The theory of membrane computing was formulated by Paun as an attempt to formulate a computational model inspired by the way in which the living cells function. P systems which is a highly distributed, parallel, theoretical model and is an area of special interest in recent times. $P$ systems have various application one such area of research is the generation of array grammars using them. In this study we define a model of $P$ system to generate a new class of languages called grammar based two-dimensional pattern languages and their picture generation.
\end{abstract}

Key Words: Two-dimensional patterns, Two-dimensional axioms, Factorization of array, Array rewriting $P$ system for grammar based $2 D$ patterns

\section{INTRODUCTION}

String grammars are used to describe various forms of language constructs, hence the study of string grammars find their application in various fields such as Linguistics, Mathematics and Computer Science. String grammars also play an important role in analysis of high level languages. In this context, a pattern ' $\alpha$ ' is a string over an alphabet set $\left\{x_{1}, x_{2}, x_{3}, \cdots\right\}$ of variables. For some finite alphabet $\Sigma$ of terminal symbols, the pattern language described by $\alpha$ (with respect to $\Sigma$ ) is the set of all words over $\Sigma$ that can be derived from $\alpha$ by uniformly substituting the variables in $\alpha$ by non-empty terminal words was introduced by Angluin [2]. Dassow et.al., [5] introduced pattern grammars which is a generative device to modify the pattern languages defined by Angluin [2] namely, not allowing the replacing of variables by arbitrary strings, but to adopt the following strategy more usual in formal language theory: start from a finite set of given strings (axioms), replace them by variables in a given set of pattern(s), all strings generated (identified) in this way constitute the associated languages. This way of obtaining languages by substituting all occurrences of the same variable by the same string is related to rewriting in parallel. In [4] the concept of pattern as language descriptors was generalized to two-dimensional case, preserving the simplicity and compactness of the descriptors.

The theory of $\mathrm{P}$ system (or membrane computing) introduced by Paun [6] is a theoretical model which is processed in a parallel manner. Recently Natural computing, a field of research which deals with the computation to imitate nature is of great interest. Already a lot of work has been done in the areas of DNA computing, Generic Algorithms and Neural networks. P systems introduced by Paun deals with how to process multi-sets by evolution rules in a hierarchical arrangement of membranes that represent the living cell. Rewriting P systems is a branch of membrane computing where objects are replaced by strings and are processed using rewriting rules.

Pictures are described as finite arrays in two-dimensional plane [3] which are connected and has been an area of great interest for many researchers. The study of pictures is in an attempt to extend recognisability in one dimension to two dimensions. Array grammars are extension of string grammars to pictures in two-dimension, where the points with integer coordinates of the plane represent symbols. It is easily seen that array grammars are immediate extensions of the string grammars, where the strings in the membranes are replaced by arrays and string operations by array rewriting rules.

In this paper we define an array rewriting $\mathrm{P}$ system to generate languages defined by two-dimensional grammars based on patterns defined in [1]. We also to analyse the picture generated by two-dimensional grammars based on patterns.

\section{PRELIMINARIES}

For a finite alphabet $\Sigma$, a string or word (over $\Sigma$ ) is a finite sequence of symbols from $\Sigma$, and $\epsilon$ stands for the empty string. The notation $\Sigma^{+}$denotes the set of all nonempty strings over $\Sigma$, and $\Sigma^{*}=\Sigma^{+} \cup\{\epsilon\}$. We say that a string $v \in \Sigma^{*}$ is a factor of a string $\omega \epsilon \Sigma^{*}$ if there are $u_{1}, u_{2} \in \Sigma^{*}$ such that $\omega=u_{1} \cdot v \cdot u_{2}$. If $u_{1}$ or $u_{2}$ is empty string then $v$ is a prefix (or a suffix respectively) of $\omega$.the notation $|\omega|$ stands for the length of a string $\omega$.

A two-dimensional word (or array) over $\Sigma$ is a tuple $\mathrm{W}=$ $\left(\left(a_{11}, a_{12}, \cdots, a_{1 n}\right),\left(a_{21}, a_{22}, \cdots, a_{2 n}\right), \cdots,\left(a_{m 1}, a_{m 2}, \cdots, a_{m n}\right)\right)$, where $\mathrm{m}, \mathrm{n} \in \mathbb{N}$ and, for every $\mathrm{i}, 1 \leq i \leq m, 1 \leq j \leq n, a_{i j} \in \Sigma$. We define width to be the number of columns and height to be the number of rows of $\mathrm{W}$. $\lambda$, denotes the empty array.For the sake of convenience, we denote $\mathrm{W}$ by $\left[a_{i j}\right]_{m, n}$ or by a matrix of one of the following form:

\footnotetext{
Revised Manuscript Received on August 05, 2019.

Christopher Kezia Parimalam, Research Scholar, Department of Mathematics, Queen Mary's College, Chennai, Tamilnadu, India (Email: keziamaths@gmail.com)

J.D.Emerald, Assistant Professor, Department of Mathematics, Queen Mary's College, Chennai, Tamilnadu, India.

(Email: emeraldsolomon96@gmail.com)
} 


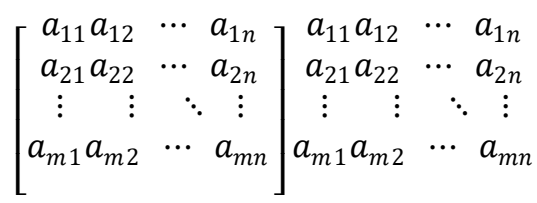

If we want to refer to the $\mathrm{j}^{\text {th }}$ symbol in row $\mathrm{i}$ of the array When we use $\mathrm{W}[\mathrm{i}, \mathrm{j}]=a_{i . j}$. By $\Sigma^{++}$, we denote the set of all nonempty arrays over $\Sigma$, and $\Sigma^{* *}=\Sigma^{++} \cup\{\lambda\}$. An array language is every subset of languages that belong to $\Sigma^{* *}$

Definition 2.1[1] Two-dimensional Pattern Grammar is a four tuple $G=(\Sigma, \Delta, \mathrm{A}, \mathrm{P})$ where

$\Sigma=$ Set of terminal alphabets

$\Delta=$ Set of pattern variables

$\mathrm{A}=$ Set of two-dimensional axioms which satisfy the patterns defined by their factorization

$\mathrm{P}=$ The two-dimensional pattern, defined by pattern variables only and some $\delta_{i j}$ the same.

$$
\text { i.e., } \mathrm{P}=\left[\begin{array}{cccc}
\delta_{11} \delta_{12} & \cdots & \delta_{1 n} \\
\delta_{21} \delta_{22} & \cdots & \delta_{2 n} \\
\vdots & \vdots & \ddots & \vdots \\
\delta_{n 1} \delta_{n 2} & \cdots & \delta_{m n}
\end{array}\right] \text { all the } \mathrm{m} x \mathrm{n} \text { entries are }
$$

defined by a pattern variable.

The pattern follows one of the concatenation (factorization) (i) row, (ii) column (iii) row-column (iv) column-row (v) proper (where it satisfies both row-column and column-row).

Given pattern $\mathrm{P}$ and initial axiom to start with $P(L)$ is the set of two-dimensional arrays obtained by replacing the variables in the pattern $\mathrm{P}$ by the arrays in $\mathrm{A}$, the same variable being replaced by the same array in all their occurrences.

The language generated by $G$, denoted by $L(G)$, is the smallest language $L \subseteq \Sigma^{* *}$ for which we have:

(i) $A \subseteq L$

(ii) $\mathrm{P}(\mathrm{L}) \subseteq L$.

Arrays are obtained starting from the axioms and using them in the patterns finite number of times. The language generated $L(G)=P \cup P(A) \cup P(P(A)) \cup P(P(P(A))) \cdots$

A language $L(G)$ thus obtained is called a twodimensional pattern Language and we denote it as

$L_{p, f}$ where $\mathrm{f}$ denotes the array factorization type of the pattern ; and its family is denoted by $\mathcal{L}_{\mathrm{P}, \mathrm{f}}$.

A two-dimensional pattern is a mxn array, and the axiom set is a set of mxn dimensional arrays which satisfy the concatenation rules for arrays either by row, column, rowcolumn or column-row or proper factorization.

Example 2.1 $G_{1}=(\{a\},\{\delta\},\{[a]\}, P), P=\left[\begin{array}{ll}\delta & \delta \\ \delta & \delta\end{array}\right]_{p}$ $L\left(G_{1}\right)=L_{P, p}$

$=A \cup$ set of all arrays of $a^{\prime} s$ with dimension $2^{n} \times 2^{n}$ where $n=1,2 \cdots$

\section{ARRAY REWRITING P SYSTEM FOR GRAMMAR BASED TWO-DIMENSIONAL PATTERN LANGUAGES}

Definition 3.1 An array-rewriting $P$ systemfor twodimensional patterns ARPS2DP (of degree $m \geq 1$ ) is a construct

$\Pi_{R}=\left(N, T, \mu, A_{1}, \cdots A_{m}, P_{1}, \cdots P_{m}, i_{0 u t}\right)$,

Where $\mathrm{N}$ is the set of pattern variables and terminals, $\mathrm{T} \subseteq V$ is the terminal alphabet, $\mu$ represents the way in which the ' $\mathrm{m}$ ' membranes are arranged in the system, $A_{1}, \cdots, A_{m}$ are the arrays present initially in the regions of $\mu$ and are finite in number, $P_{1}, \cdots P_{m}$, are finite set of rewriting rules in the $\mathrm{m}$ regions of $\mu$; the pattern rewriting rules are of the form $A \rightarrow B_{(t a r)}$; where the target can be in, out, here, $A, B \in(N \cup T)^{*}, i_{0 u t}$ is the label of the output membrane.

A computation in an array rewriting $\mathrm{P}$ system for $2 \mathrm{D}$ patterns is defined in the same way as in a string rewriting $P$ system. Each variable in the array from each region can be rewritten by exactly one rewriting rule applicable to it in the given region, the same variable being rewritten with the same rule and is sent to the target specified in the rewriting rule. When there are no more rewriting rule to be applied and if the array reaches the output membrane the computation stops. A successful computation consists of arrays composed only of symbols from $\mathrm{T}$ placed in the membrane with label $i_{0 u t}$ in the halting configuration. The set of all such arrays generated by the system $\Pi_{R}$ is denoted by $\operatorname{APL}\left(\Pi_{R}\right)$.

We now give an example of an array rewriting $\mathrm{P}$ system that can generate a given two-dimensional pattern language based on grammars that defines it. We consider the case where the pattern is a two-dimensional array in particular a $2 \times 2$ array and the axioms are over a single alphabet.

Example 3.1 Consider the two-dimensional pattern grammar $G_{2}=(\Sigma, \Delta, A, P)$

Where, $\quad \Sigma=\{a, b\} ; \quad \Delta=\left\{\delta_{1}, \delta_{2}\right\} ; A=\{a, b\}$;

$L\left(G_{2}\right)$

$=\left\{\left[\begin{array}{ll}w_{1} & w_{2} \\ w_{1} & w_{2}\end{array}\right] / w_{1}, w_{2}\right.$ are square matrices of order $2^{m}$ $\left.\times 2^{m}, m=0,1, \cdots\right\}$

The array rewriting $\mathrm{P}$ system to generate the above twodimensional pattern language is defined as follows: $\Pi_{R 2}=$ $\left(N, T, A_{1} \cdots A_{3}, P_{1} \cdots P_{3}, i_{1}\right)$; where

$$
\begin{aligned}
& \mu=\left[{ }_{1}\left[{ }_{2}[3]_{1}\right]_{2}\right]_{3} \\
& \left.A_{1}=\left\{a, b, \begin{array}{ll}
\eta_{11} & \eta_{22} \\
\eta_{11} & \eta_{22}
\end{array}\right\} ; A_{2}=\phi ; A_{3}=\begin{array}{ll}
\eta_{11} & \eta_{22} \\
\eta_{11} & \eta_{22}
\end{array}\right\} \\
& P_{1} \\
& =\left\{\begin{aligned}
\eta_{11} \rightarrow a, \eta_{11} \rightarrow b, \eta_{12} & \rightarrow a, \eta_{12} \rightarrow b, \eta_{21} \rightarrow a, \eta_{21} \rightarrow b, \\
\eta_{22} & \rightarrow a, \eta_{22} \rightarrow b
\end{aligned}\right\}
\end{aligned}
$$

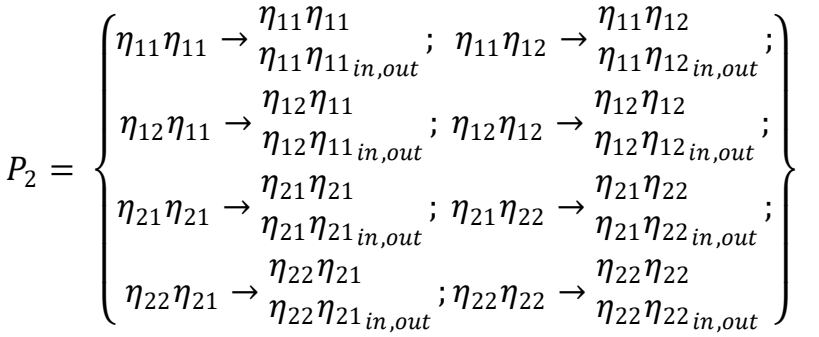


$P_{3}=\left\{\begin{array}{c}\eta_{11} \rightarrow \eta_{11} \eta_{11_{\text {in }}} / \eta_{11} \eta_{12} / \eta_{12} \eta_{11_{\text {in }}} / \eta_{12_{\text {in }}} ; \\ \eta_{12} \rightarrow \eta_{11} \eta_{11_{\text {in }}} / \eta_{11} \eta_{12_{\text {in }}} / \eta_{12} \eta_{11_{\text {in }}} / \eta_{12_{\text {in }}} \\ \eta_{21} \rightarrow \eta_{21} \eta_{21_{\text {in }}} / \eta_{21} \eta_{22_{\text {in }}} / \eta_{22} \eta_{21_{\text {in }}} / \eta_{22} \eta_{22_{\text {in }}} \\ \eta_{22} \rightarrow \eta_{21} \eta_{21_{\text {in }}} / \eta_{21} \eta_{22_{\text {in }}} / \eta_{22} \eta_{21_{\text {in }}} / \eta_{22} \eta_{22} \text { in }\end{array}\right\}$

$\mathrm{P}=\left(\boldsymbol{D}_{2}\right) \ominus\left(\delta_{1} \delta_{2}\right) ;$ Dce we have a rule in the third membrane which first grows each pattern variable according to the column catenation rule in the first parenthesis of the pattern rule and the second membrane has a rule to grow the pattern according to the row catenation rule. Thus we form the frame work to grow the patterns and finally terminate them using the termination rules in the first membrane which is the output membrane.

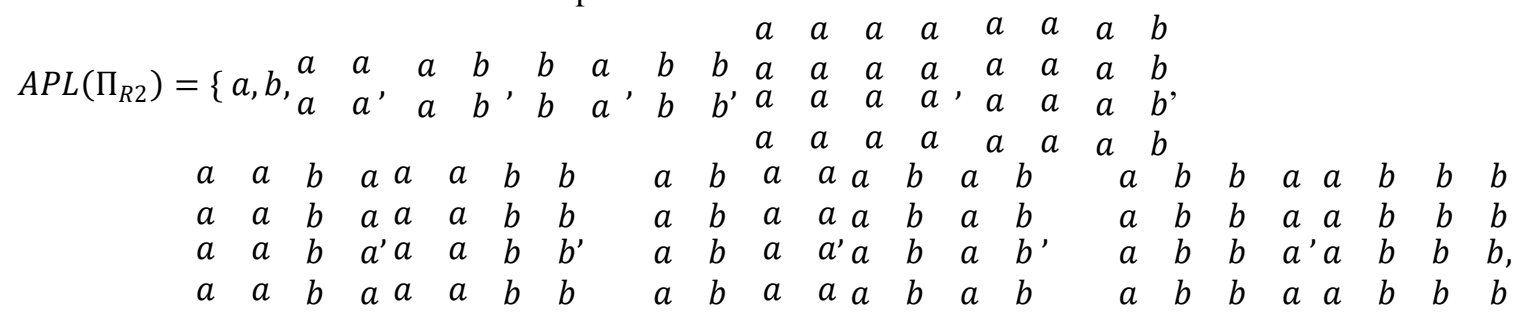

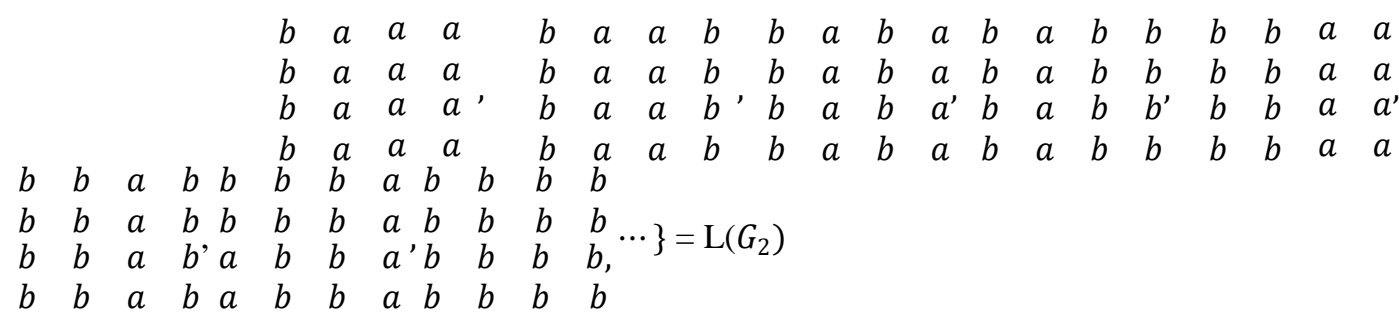

\section{CONSTRUCTION OF AN ARRAY REWRITING P SYSTEM MODEL FOR TWO-DIMENSIONAL PATTERNS}

Theorem 4.1 For every two-dimensional grammar based on pattern we have an Array Rewriting P System for twodimensional pattern $A R P S 2 D P_{3}$ that generates it.

Proof: We give the construct for the Array rewriting $\mathrm{P}$ system for two-dimensional pattern in two cases. The first being the $2 \mathrm{D}$ pattern satisfying column-row factorization and the second when the $2 \mathrm{D}$ pattern satisfies the rowcolumn factorization.

Case 1: Given a 2D pattern grammar $G=(\Sigma, \Delta, \mathrm{A}, \mathrm{P})$, in which the $2 \mathrm{D}$ pattern satisfies the column-row factorization, we define the P-system model where

$$
\Pi_{R}=\left(N, T, \mu, A_{1}, \cdots A_{3}, P_{1}, \cdots P_{3}, i_{1}\right)
$$

$N=\left\{\begin{array}{r}\eta_{i j}, i=1,2 \cdots n \text {, for every } \delta_{i} \in \Delta, i=1 \cdots n ; \\ j=1 \cdots\end{array}\right.$

$T=A$

$\mu=\left[{ }_{1}\left[{ }_{2}[3]_{3}\right]_{2}\right]_{1}$

$A_{1}=\{A, P\}$; where $\mathrm{P}$ is replaced with the $\eta_{i i}$ in the place of $\delta_{i}$.

$A_{2}=\phi ; A_{3}=P$ as defined in $A_{1}$

$R_{1}=\left\{\eta_{i j \rightarrow} a_{t}\right.$, for $i,=1, \cdots n, j=1, \cdots m$, for $\quad$ each $\left.a_{t} \in A\right\}$

$R_{2}$
$=\left\{\begin{array}{c}{\left[\eta_{i 1} \cdots \eta_{i s}\right]^{T} \rightarrow\left[\eta_{i 1} \cdots \eta_{i t}\right]^{T} ; \text { if }\left[\delta_{1} \cdots \delta_{s}\right]^{T} \rightarrow\left[\delta_{1} \cdots \delta_{t}\right]^{T}} \\ \text { is a row catenation rule } \\ \text { in the given } 2 D \text { pattern for the } i^{\text {th }} \text { column and } \eta_{\text {in }}=\eta_{\text {im }} \text { if } \delta_{n}=\delta_{m}\end{array}\right\}$ in,out

$R_{3}=\left\{\eta_{i i} \rightarrow \eta_{i 1} \cdots \eta_{\text {in }}\right.$; for $i$

$=1 \cdots n$, if $\delta_{1} \cdots \delta_{n}$ is the first row of the $2 D$ pattern; and $\eta_{i j} \rightarrow$

$\eta_{i 1} \cdots \eta_{\text {in }}$ for every $\eta_{i j}$ on the RHS of the productions of $\eta_{i i}$ \}
To begin with the $\mathrm{P}$ system contains the initial words in membrane 1 and 3 . The set of axioms are present in membrane 1 which are the initial words in the pattern language. The pattern is present at the beginning in membrane 1 and 3. Applying the rules of $R_{1}$ to the pattern variables in membrane 1 we get the resultant as $\mathrm{P}(\mathrm{A})$. In membrane three applying the rules of $R_{3}$, each pattern variable expands in the column where each variable is expanded to form the first row of the array in $\mathrm{P}(\mathrm{P}(\mathrm{A}))$ and sent into membrane 2. In this membrane the pattern is expanded according to the row catenation rules in the pattern and is sent to membrane 1 and 3 . In membrane 1 the terminating rules are applied to the pattern variables which result in $\mathrm{P}(\mathrm{P}(\mathrm{A}))$. While the membrane 3 further builds the array in column and then again sends it membrane two where the process is repeated and the resultant is $\mathrm{P}(\mathrm{P}(\mathrm{P}(\mathrm{A})))$. Thus $A R P S 2 D P_{3}$ generates $L(\mathrm{G})$.

Case 2: We now give the construction for $2 \mathrm{D}$ patterns which satisfy row-column factorization rule. $\mathrm{T}, \mu$, $A_{1}, A_{2}, A_{3}$ are as defined in case1.

$N$

$=\left\{\begin{array}{c}\eta_{i j}, i=1,2 \cdots n, \text { for every } \delta_{i} \in \Delta, i=1 \cdots n ; \\ j=1 \cdots m \text {, where } m \text { is the number of columns in the pattern }\end{array}\right\}$

The array rewriting rules for the three membranes are defined as follows

$R_{1}=\left\{\eta_{i j \rightarrow} a_{t}\right.$, for $i,=1, \cdots n, j=1, \cdots m, \quad$ for $\quad$ each $\left.a_{t} \in A\right\}$

$R_{2}=$

$\left\{\begin{array}{c}\eta_{i 1} \cdots \eta_{i s} \rightarrow \eta_{i 1} \cdots \eta_{i t} ; \text { if } \delta_{1} \cdots \delta_{s} \rightarrow \delta_{1} \cdots \delta_{t} \\ \text { is a column catenation rule } \\ \text { in the given 2D pattern for the } i^{\text {th }} \text { row and } \eta_{i n}=\eta_{i m} \text { if } \delta_{n}=\delta_{m}\end{array}\right\}_{\text {in out }}$ 
$R_{3}=$

$\left\{\eta_{i i} \rightarrow\left[\eta_{i 1} \cdots \eta_{\text {in }}\right]^{T}\right.$ foreach $i=1 \cdots n$,

if $\left[\delta_{1} \cdots \delta_{n}\right]^{T}$ is the first column of the $2 D$ pattern; and $\eta_{i j} \rightarrow$

$$
\begin{gathered}
G_{3}=\left(\{a, b\},\left\{\delta_{1}, \delta_{2}\right\},\left\{\left[\begin{array}{ll}
a & a
\end{array}\right],\left[\begin{array}{ll}
b & b \\
b & b
\end{array}\right], P\right\}\right), P= \\
{\left[\begin{array}{ll}
\delta_{1} & \delta_{2} \\
\delta_{2} & \delta_{1}
\end{array}\right]_{r c} \text {; i.e., } \mathrm{P}=\left(\delta_{1} \ominus \delta_{2}\right)\left(\delta_{2} \ominus \delta_{1}\right)}
\end{gathered}
$$

$\left[\eta_{i 1} \cdots \eta_{i n}\right]^{T}$ for every $\eta_{i j}$ on the RHS of the productions of $\left.\operatorname{Lr} \eta_{i} G_{3}\right)=\mathcal{L}_{p, r c}=\left[\begin{array}{ll}w_{i} & w_{j} \\ w_{j} & w_{i}\end{array}\right]$ \} .

$$
=A \cup \text { set of all arrays of dimension }
$$

$\left(\mathrm{sm}_{1}+\mathrm{lm}_{2}\right) \times 2$ knover the given axioms.

The working is similar as in case 1 , and we see that the $\mathrm{P}$ system generates $\mathrm{L}(\mathrm{G})$.

A 2D pattern defined by proper factorization can be generated either by $A R P S 2 D P_{3}$ in case 1 or

case 2.

Thus $A R P S 2 D P_{3}=\mathrm{L}(\mathrm{G})$.

Example4.1

The $A R P S 2 D P_{3}$ to generate the $G_{3}$ is defined by

$\Pi_{R 3}=\left(N, T, \mu, A_{1}, A_{2}, A_{3}, P_{1}, P_{2}, P_{3}, i_{1}\right)$

$N=\left\{\eta_{11}, \eta_{12}, \eta_{21}, \eta_{22},\right\}$

$T=\{a, b\}$

$\mu=\left[{ }_{1}\left[{ }_{2}\left[{ }_{3}\right]_{1}\right]_{2}\right]_{3}$,

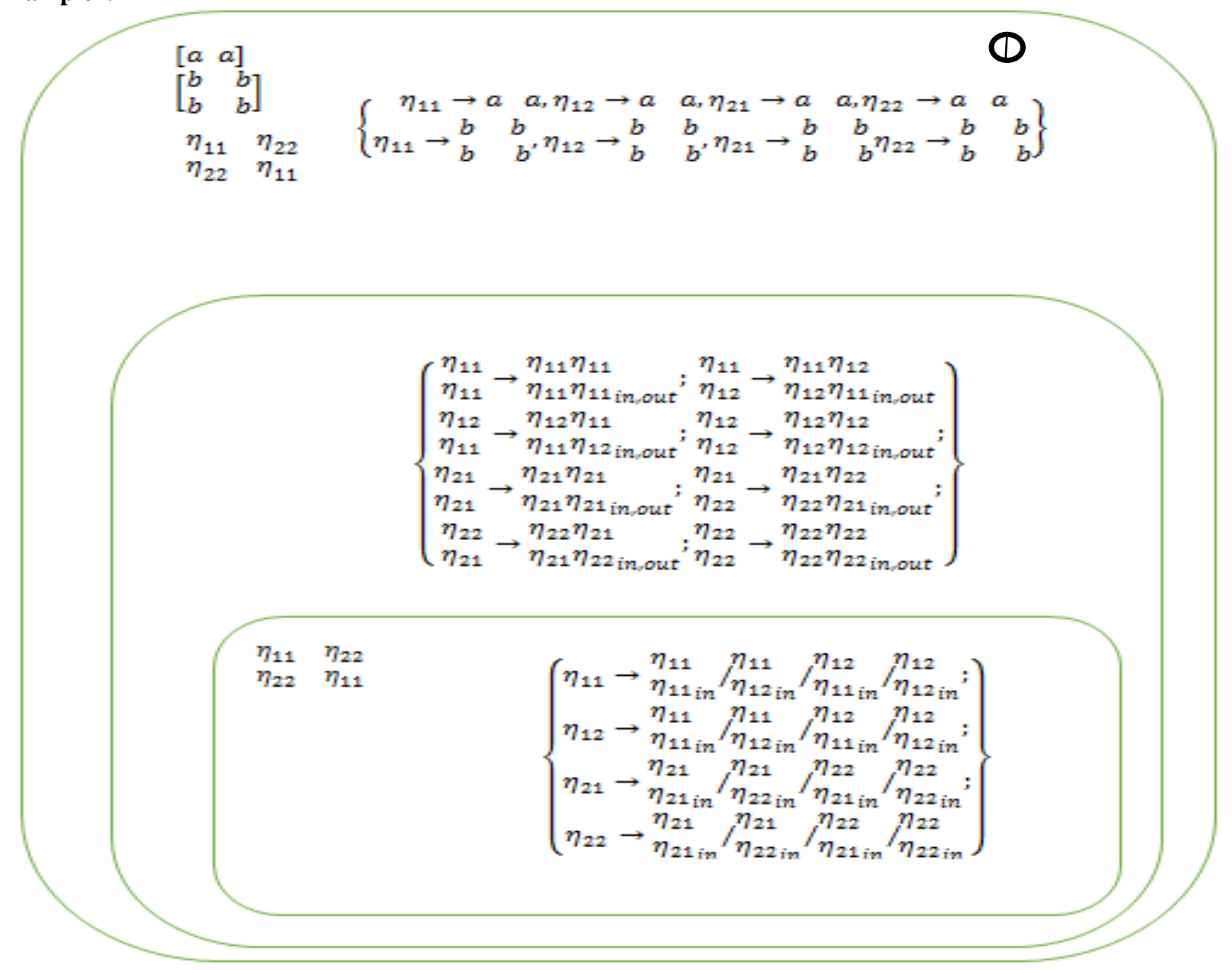

Fig 1 Array Rewriting P System generating the 2D Pattern Language

\section{PICTURE GENERATION FOR TWO- DIMENSIONAL PATTERN LANGUAGES\& RESULTS}

In this section, we generate pictures for the grammar based two-dimensional pattern languages defined above using pasting rules in Array rewriting $\mathrm{P}$ system for 2D pattern $A R P S 2 D P_{3}$. Consider the example 3.1, we associate two tiles for the two axioms, namely ' $a$ ' a hexagon and ' $b$ ' a rhombus. The ARPS $2 D P_{3}$ with pasting rules to generate the pictures associated with the $2 \mathrm{D}$ pattern language is given in Fig 2.

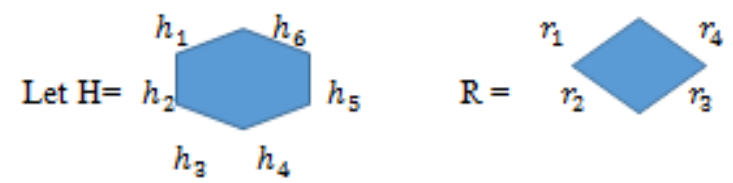

$h_{3} \quad h_{4}$ 


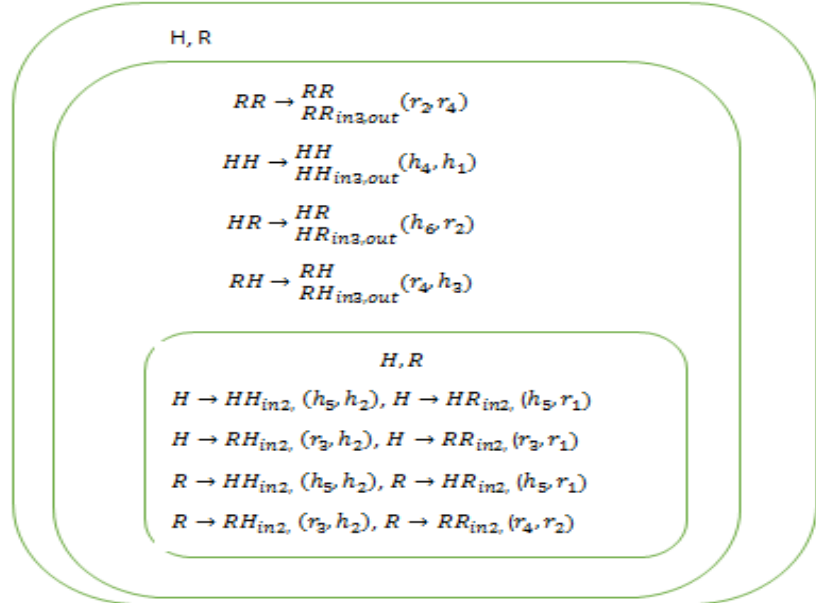

Fig $2 A R P S 2 D P_{3}$ with pasting rules for picture generation
The $\mathrm{P}$ system initially contains the hexagon and the rhombus which are the axioms in the two-dimensional language. The second membrane is empty with no initial words, hence no rules can be applied at thi stage in this membrane. The third membrane has the hexagon and the rhombus and the one among the four rules is applied. Suppose if the first rule is applied then the resultant would be two hexagons pasted with their edges $h_{5}, h_{2}$ pasted together and pushed into membrane 2. In membrane two the first rule is used and the resultant is pushed into membrane three and membrane 1 which is the output membrane.

In membrane 3, the pastirg rule

In membrane 2, the pasting is

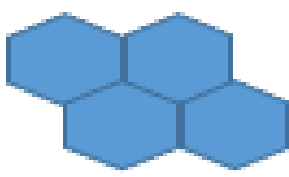

and is the output which belongs to $\mathrm{P}(\mathrm{A})$.

The next output in the series is

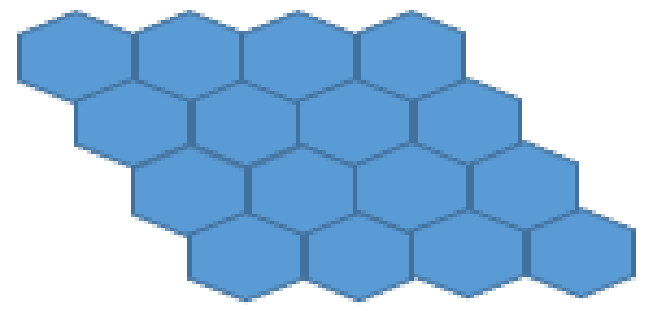

belongs to $\mathrm{P}(\mathrm{P}(\mathrm{A})$ )

Applying the zecond rule in both membranes the first, aecond and third output will be

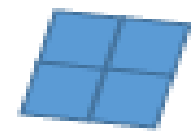

$\mathrm{P}(\mathrm{A})$

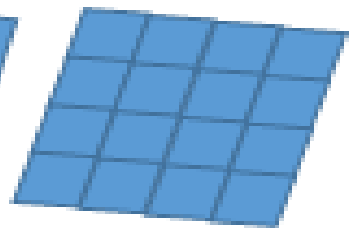

$\mathrm{P}(\mathrm{P}(\mathrm{A}))$

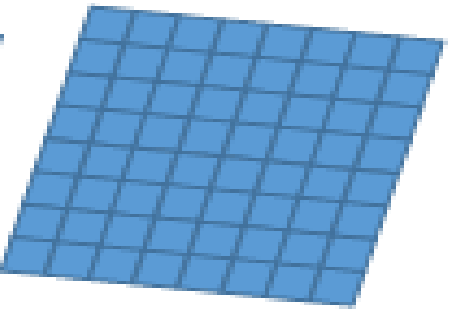

$P(P(P(A))$

Some of the outputs for P(A) when other rules are applied in membrane three is given below
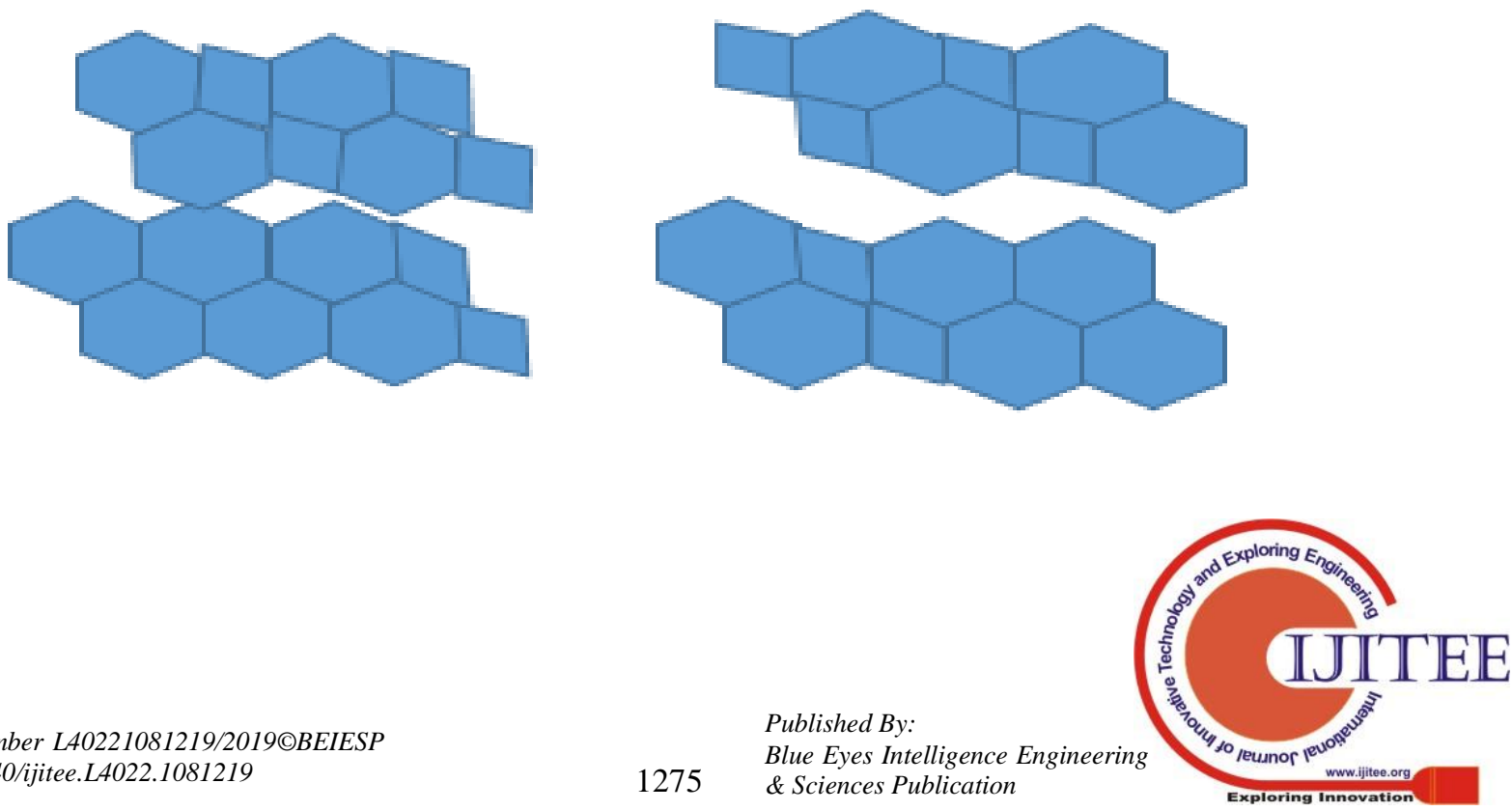


\section{CONCLUSION}

In this paper we have defined an array rewriting $\mathrm{P}$ system model to generate two-dimensional pattern languages based on pattern grammar with two-dimensional axioms. We also generated pictures associated with these languages. The study would be further extended to make a comparative study of 2D pattern languages with other array languages and picture languages.

\section{REFERENCES}

1 C.K. Parimalam, J.D. Emerald Two-dimensional grammars based on Patterns, International Journal for Computer Science and Engineering,Vol.07, Issue.05, pp.216-220, 2019.

2 D. Angluin, Finding Pattern common to set of strings, Journal of Computer and System Sciences 21, 46-62, 1980.

3 3. D. Giammarresi, A. Restivo, Recognizable picture languages, International Journal of Pattern Recognition and Artificial Intelligence. 6:31-46, 1992.

4 H. Fernau, M.L. Schmid, K.G. Subramanian, TwoDimensional Pattern Languages, In S.Bensch, F. Drewes, R. Freund, and F.Otto, editors, Fifth Workshop on NonClassical Models for Automata and Applications, NCMA, Volume 294 of books@ocg.at, pp.117-132. Osterreichische Computer Gesellschaft, 2013.

5 J. Dassow, Gh. Paun, Arto Salomaa, Grammars based on Patterns, International Journal of Foundations of Computer Science, Vol 4: 1-14, 1993.

6 Gh. Paun , Computiong with Membranes, Journal of Computer and System Sciences,61,108- 143, 2000. 